\title{
Capsule Commentary on Brenner et al., Comparing 3 Values Clarification Methods for Colorectal Cancer Screening Decision-Making: A Randomized Trial in the US and Australia
}

\author{
J. S. Blumenthal-Barby, PhD, MA \\ Center for Medical Ethics and Health Policy, Baylor College of Medicine, Houston, TX, USA.
}

J Gen Intern Med 29(3):519

DOI: $10.1007 / \mathrm{s} 11606-013-2741-5$

(c) Society of General Internal Medicine 2013

$\mathrm{T}$ his study investigated whether different types of "values clarification exercises - or methods" (VCMs) impacted (a) what attribute patients named as most important in making decisions about colorectal cancer screening, and (b) their decisions about colorectal screening (whether to get screened and which type of test to have). They found that the different VCMs did not produce any difference in terms of decisions about colorectal screening, but argue that they did impact which attribute patients named as most important. ${ }^{1}$

This is a very important study, since, as the authors note, most decision aids have some sort of section that helps a patient think about and compare the desirability of the options (oddly, termed a "values" clarification exercise).

While it is true that there is a sense in which the different VCMs had an impact on which attributes patients designated as most important to them, there is a more important sense in which the VCMs did not. The sense in which they did, which the authors report, is that those who went through the VCM of ranking [abstract] attributes were more likely to choose "reduction in risk" as more important than those who went through the two other VCMs. The sense in which the type of VCM did not impact which attributes patients thought most important, however, is that the ordering of the importance of the attributes was actually exactly the same (with one minor exception) across all VCMs. "Risk reduction" was always the most important attribute, followed by "nature of the test," "frequency of the test," "chance of complications," and "chance of needing a colonoscopy."

The authors conclude that no meaningful differences in test preference (whether to do it and what type) emerged based on the VCM, but this may be partially because the VCMs tested contained verbiage that was rather abstract (e.g., attribute of "nature of the test" was just described as, say, mild discomfort, invasive, done in a hospital). VCMs with more concrete descriptions of the "nature" of a colonoscopy may have a significant impact on patient choices. The essential message is that VCMs are potentially very powerful exercises for shaping patients' decisions.

Conflict of Interest: The author has no conflict with any of the material in this manuscript.

Corresponding Author: J. S. Blumenthal-Barby, $\mathrm{PhD}$, MA; Center for Medical Ethics and Health Policy, Baylor College of Medicine, Houston, TX, USA (e-mail: jsswinde@bcm.edu).

\section{REFERENCES}

1. Brenner A, Howard $\mathbf{K}$, Lewis $\mathbf{C}$, et al. Comparing 3 Values Clarification Methods for Colorectal Cancer Screening Decision-Making: A Randomized Trial in the US and Australia. J Gen Intern Med. 2013. doi:10.1007/ s11606-013-2701-0. 